\title{
Comparative study between conventional ultrasound strain elastography and an Al-enabled elastography software in differentiating breast masses
}

Eman Faker Kamal ${ }^{1}$, Rasha Mohamed Kamal ${ }^{1}$, Ahmed M. Mahmoud ${ }^{2,3}$, Mahmoud Ibrahim Mekhaimar ${ }^{1}$ and Mennatallah Mohamed Hanafy ${ }^{1 *}$ (])

\begin{abstract}
Background: Conventional ultrasound elastography is a relatively novel non-invasive imaging study that assesses tissue stiffness and helps in the characterization of breast lesions. However, strain elastography is not available in some ultrasound machines especially those before 2003 and issusceptible by motion artifacts. Our aim was to compare the results of conventional ultrasound elastography and the results of an Advanced intelligence enabled elastography software. Also, we aimed to assess the feasibility of the Al-enabled elastography software to overcome the unavailability of the conventional elastography software in some new ultrasound machines.

Results: The study included 53 patients, who had breast lesions either clinically felt or detected during screening. All patients were subjected to both grayscale US imaging and conventional ultrasound elastography; quasi-static compression was applied during acquiring one of the cine-loops of the greyscale US imaging. Also,the Cine-Loops of the grayscale US imaging while quasi-static compression were processed by an Al-enabled elastography software. Then the results of the strain ratio (SR) calculated by conventional elastography software and those by Al-enabled elastography software were compared. The strain ratio calculated using the Al-enabled elastography software showed better results than conventional ultrasound elastographys train ratio. The Al-enabled software shows better specificity, sensitivity, positive predictive values, and negative predictive values than the conventional ultrasound elastography.

Conclusion: The Al-enabled elastography software shows promising results compared to the conventional US elastography. Elastography does not have the potential to replace conventional B-mode US for the detection of breast cancer but may complement the conventional US to improve diagnostic performance.
\end{abstract}

Keywords: Elastography, Strain ratio, Breast cancer

\section{Background}

Breast cancer is the most common malignancy in women worldwide and a leading cause of death. It accounts for $25 \%$ of all cancers and $15 \%$ of all cancer deaths among

\footnotetext{
*Correspondence: drmennamhanafy@gmail.com

1 Department of Diagnostic and Interventional Radiology, Faculty of Medicine, Cairo University, 1 kasr Elainy street Fom El kalig, 52 Tayaran Street, Nasr City Cairo, Nasr City 00202, Egypt

Full list of author information is available at the end of the article
}

women [1]. Clinically, palpation plays a fundamental role in the detection and evaluation of any breast lesion where malignant lesions are usually hard than normal and benign lesions [2].

Elastography is a complementary imaging technique to Ultrasound that can be used to assess tissue stiffness thus improving the diagnosis of breast cancer and avoiding unnecessary breast biopsies $[2,3]$. 
Elastography assessment has been added to ACR BIRADS atlas fifth edition where lesions are classified into soft, intermediate and hard. Malignant breast lesions are usually stiff compared to benign lesions which are usually soft and elastic $[4,5]$.

The two most frequently used elastographic techniques are strain and shear wave elastography. For strain elastography, a qualitative visual analysis of the color pattern using the Tsukuba Score classification and the semiquantitative strain ratio are the usual methods used for classification and interpretation [6].

Although some ultrasound companies have developed elastography on some of their premium machines, it is still unavailable in other machines. Additionally, the output of elastography imaging may vary from a manufacturer to another due to the different algorithms and platforms $[6,7]$.

AI-enabled Elastography Software can provide a solution to overcome the unavailability of elastography in old systems and the cost of new devices with elastography on their premium machines [8]. It was proven to reconstruct ultrasound strain elastography from conventional grayscale ultrasound images acquired by commercially available ultrasound machines regardless of the model and manufacturer [9].

Our aim was to compare the results of conventional US elastography with those results corresponding to the AIenabled elastography software. Also, we aimed to assess the feasibility of using the AI-enabled elastography software to overcome the unavailability of the elastography software in some ultrasound machines.

\section{Methods}

The study was a prospective study carried over nine months with additional 18 months (3 follow-up studies every 6 months) when close follow-up was requested to confirm the diagnosis in some of the cases. This study included 53 patients. All patients had breast lesions either clinically felt or detected during screening. Three patients presented with pain in addition to the mass. All patients were subjected to grayscale ultrasound examination. Elastography strain ratio was evaluated by both methods; once using the conventional ultrasound elastography software and once by the advanced intelligent elastography software. Results of both methods were compared together in reference to the final diagnosis.

The final diagnosis was correlated either by histopathological analysis of biopsy samples (for solid lesions encountered 35), or cytological analysis via fine-needle aspiration in cystic lesions. Close follow-up (for 18 benign lesions assigned BIRADS 2) was considered in the study. All were correlated according to the BIRADS lexicon).
The study was approved by the ethical committee, and informed written consent was taken from all subjects.

Grey-scale ultrasonography and conventional Ultrasound elastography were performed by two sonographers, one specialist with 2 years experience in breast US and elastography was repeated as well as the interventional procedures were performed by breast imaging consultant with 14 years experience in breast US and interventional breast procedures.

The AI-elastography software images were processed using the software postulated by the biomedical imaging crew, which attained 3 years experience in their field.

\section{Subjects \\ Inclusion criteria}

- Patients with non-skin breast lesions.

\section{Exclusion criteria}

- Metastatic masses with known primary, recurrence or invasion from nearby tumor e.g. rib chondrosarcoma.

- Patients with generalized edema due to heart/liver/ renal failure or lymphatic edema due to axillary lymph node clearance.

\section{Conventional ultrasound elastography technique}

- Conventional ultrasound scan and conventional strain elastography had been done on all patients using General Electric Logic S8 and/or General Electric (GE) Logic P7 both with a linear probe of 7.5$12 \mathrm{MHz}$ frequency.

- The patient lied supine with the ipsilateral arm is raised over the patient's head. Gel was applied to the examined breast. The probe was perpendicular to the skin. A good quality B-mode image was obtained. A region of interest (ROI) larger than lesion including fatty breast tissue was used. The (ROI) was adjusted to place the suspicious lesion at the center. Slight steady compressions were done for about $5 \mathrm{~s}$ duration. Where the accuracy of the technique was evaluated on a quality bar indicator. The results were displayed as a color-coded map. The strain ratio was obtained between two measurements; the first measurement was drawn at the suspicious lesion and the second at the reference tissue where the two areas were approximately at the same depth and size. 


\section{Advanced intelligent elastography software technique}

- Cine-loops of breast elastography were collected while applying freehand compression (1-5\%) using the transducer. Compressions should be slow and steady, rapid compressions may distort the images and yet leading to inconvenient resuls. Cine-loops of conventional gray-scale images were then imported to a vendor-neutral AI-enabled elastography software (US-Elasto, Dileny Technologies and biomedical engineering LLC, Giza, Egypt). The software adopts a novelpatented method to reconstruct strain elastography from conventional gray-scale images after removing motion artifacts [10]. The Frame-toframe displacements were calculated using hierarchy recursive displacement tracking technique. At least 5 slow and firm compressions were included in the cine-loops. The strain was calculated as displacement spatial derivative and was then superimposed on gray-scale images to provide both anatomical and mechanical information.

\section{Statistical analysis}

Data analysis packages were SPSS version 21 and Epi calc Qualitative data was presented by number and percentage, quantitative data was presented by mean, standard deviation, median, and range. Statistical tests done to detect sensitivity, specificity, positive predictive value and negative predictive value, positive like hood ratio, and negative like hood ratio. Correlation was done using Pearson correlation coefficient and the level of significance was considered equal to or below 0.05 .

Receiver-operating characteristic (ROC) curve analysis was used to determine the cut off value for Strain ratio. The cut off value for strain ration was calculated by both conventional elastography and Advanced intelligent elastography software where it was the point in which sensitivity equals specificity by either elastography.

\section{Results}

Our study included 53 cases with 53 breast lesions. The ages ranged from $29-71$ years with a mean age of $49.298 \pm 10.75$ (mean \pm SD). Upon correlating with the final diagnosis either by histological analysis of tissue samples or postoperative pathology (for 35 lesions), cytological analysis, or close follow up (applied on 18 lesions that were categorized as BI-RADS 2 and showed stationary course for 1 year in a way to confirm their benign nature according to the BIRADS lexicon); 25/53 (47.2\%) lesions were benign while 28/53 (52.8\%) were malignant.

According to Ultrasound findings, 20/53 (37.7\%) lesions were considered benign (assigned BIRADS 2 and 3 categories) while 33/53 (62.3\%) lesions were considered malignant (assigned BIRADS 4 and 5). According to the histopathology results or close follow-up; 23 lesions were true positives, 10 were false positives, 5 lesions were false negatives and 15 were true negatives. The calculated ultrasound sensitivity, specificity, positive likelihood, negative likelihood, positive predictive value, and negative predictive value were $60 \%, 82.14 \%, 2.05,0.3,69.7 \%$, and $75 \%$ respectively (Table 1 ).

The Strain ratio was calculated once by conventional elastography and once by the advanced intelligent elastography software.

The cut-off point for Strain ratio calculated by conventional elastography was 2.4 and accordingly, 28 153 (52.8\%) lesions were considered benign (assigned BIRADS 2 and 3 categories) while $25 / 53$ (47.2\%) lesions were considered malignant (assigned BIRADS 4 and 5). According to the histopathology results or close followup;13 lesions were true positives, 12 were false positives, 15 lesions were false negatives and 13 were true negatives. The calculated Conventional elastography strain ratio sensitivity, specificity, positive likelihood, negative likelihood, positive predictive value, and negative predictive value, were $46.43 \%, 52 \%, 0.97,1.03,52 \%, 46.43 \%$ respectively (Table 1).

The cut-off point for Strain ratio calculated by Advanced intelligent elastography software was 2.28

Table 1 Comparison of Ultrasound, Conventional elastography, and advanced intelligent software diagnostic indices

\begin{tabular}{llll}
\hline & Ultrasound & $\begin{array}{l}\text { Strain ratio calculated by conventional } \\
\text { elastography }\end{array}$ & $\begin{array}{l}\text { Strain ratio calculated by } \\
\text { Advanced intelligent elastography } \\
\text { software }\end{array}$ \\
\hline Sensitivity & $60 \%$ & $46.43 \%$ & $60.71 \%$ \\
Specificity & $82.14 \%$ & $52 \%$ & $64 \%$ \\
Positive likelihood ratio & 2.05 & 0.97 & 1.6 \\
Negative likelihood ratio & 0.3 & 1.03 & 0.6 \\
PPV & $69.7 \%$ & $52 \%$ & $65.4 \%$ \\
NPV & $75 \%$ & $46.43 \%$ & $59.3 \%$ \\
\hline
\end{tabular}


and accordingly, 27 /53 (50.9\%) lesions were considered benign (assigned BIRADS 2 and 3 categories) while 26 I53 (49.1\%) lesions were considered malignant (assigned BIRADS 4 and 5). According to the histopathology results or close follow-up;17 lesions were true positives, 9 were false positives, 11 lesions were false negatives and 16 were true negatives. The calculated Advanced intelligent elastography software strain ratio sensitivity, specificity, positive likelihood, negative likelihood, positive predictive value, and negative predictive value were $60.71 \%, 64 \%, 1.6,0.6,65.4 \%, 59.3 \%$ respectively (Table 1 ).

Correlation between conventional elastography software and advanced intelligent elastography software was assessed as shown in Fig. 1.

\section{Discussion}

Breast cancer is the most common cancer worldwide in females around World [11].

Breast ultrasound is a non-invasive, relatively available imaging modality that is used as complementary to mammography in screening especially in dense breasts, differentiation of solid and cystic lesions, characterization of lesions, and diagnosis of intraductal mass as well as problem-solving for mammographically detected asymmetries $[12,13]$.

US strain elastography is a semiquantitative imaging study that offers the ability to visualize the strain produced from applying pressure to the breast tissue. The strain is smaller in harder tissue than in softer tissue [6].

Conventional elastography software is still not available in some new ultrasound machines. Advanced intelligent elastography software can provide a solution to overcome the unavailability of elastography in ultrasound machines $[7,8]$.

This study aimed to compare the results of conventional ultrasound elastography and the results of advanced intelligent elastography software as well as to assess the feasibility of using the Advanced intelligent elastography software to overcome the unavailability of the conventional elastography software in some new Ultrasound machines.

The current study included 53 patients. Upon correlation with final diagnosis either by pathological analysis of tissues samples (for 35 lesions), or close follow-up (applied for 2 years for 18 lesions assigned BIRADS 2). Twenty-five out of fifty-three lesions were benign (47.2\%) and twenty eight out of fifty-three (52.8\%) lesions were malignant.

We assessed the sensitivity and specificity of the conventional US compared to the pathological results and/or close follow-up.

In the current study, we reported a sensitivity of about $60 \%$ and a specificity of about $82.14 \%$.

In our study, we calculated conventional SR for each lesion (Figs. 2,3). According to the histopathology results

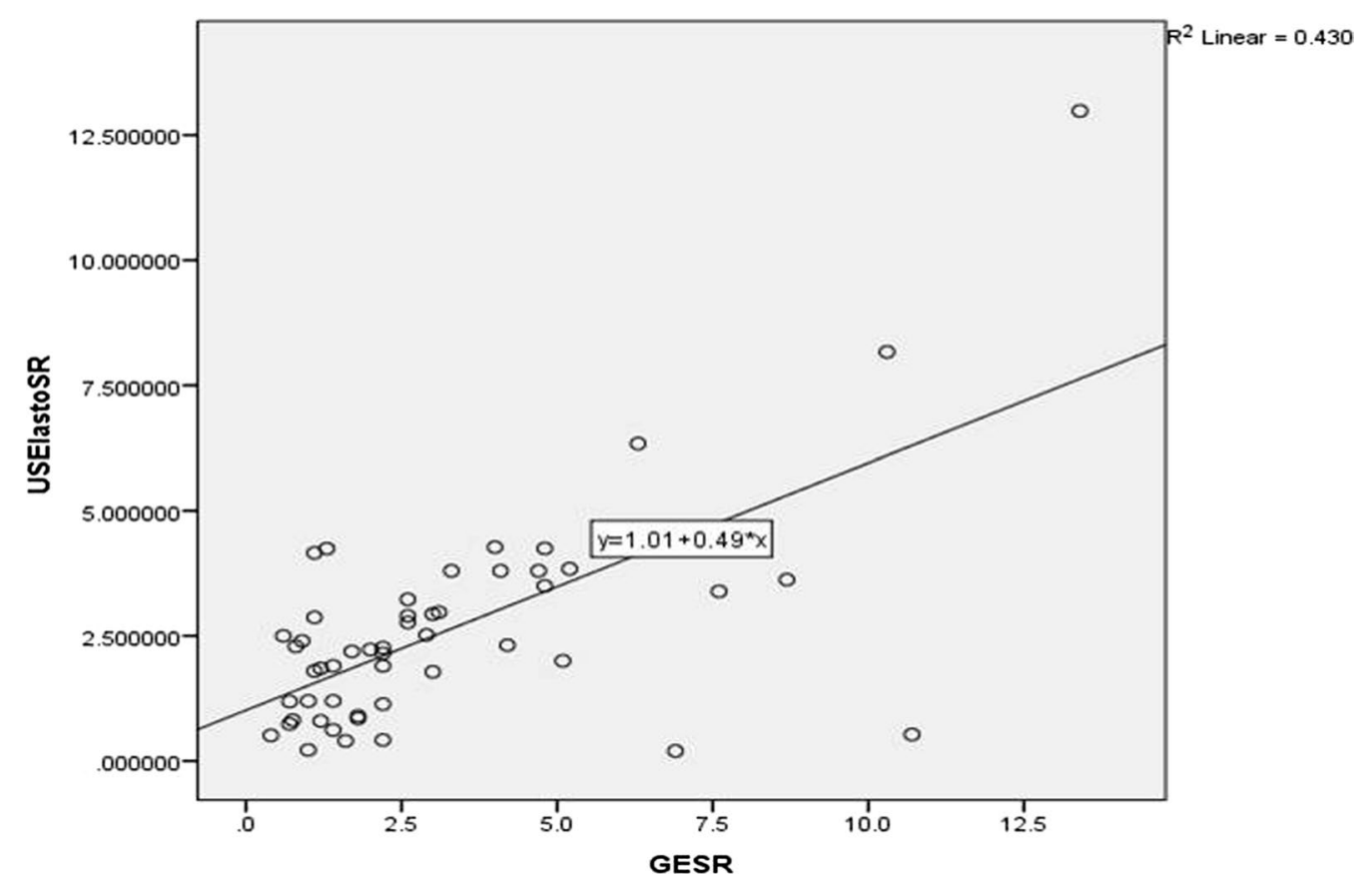

Fig. 1 Moderate correlation between the SR calculated by the conventional elastography software and Al-enabled elastography software 

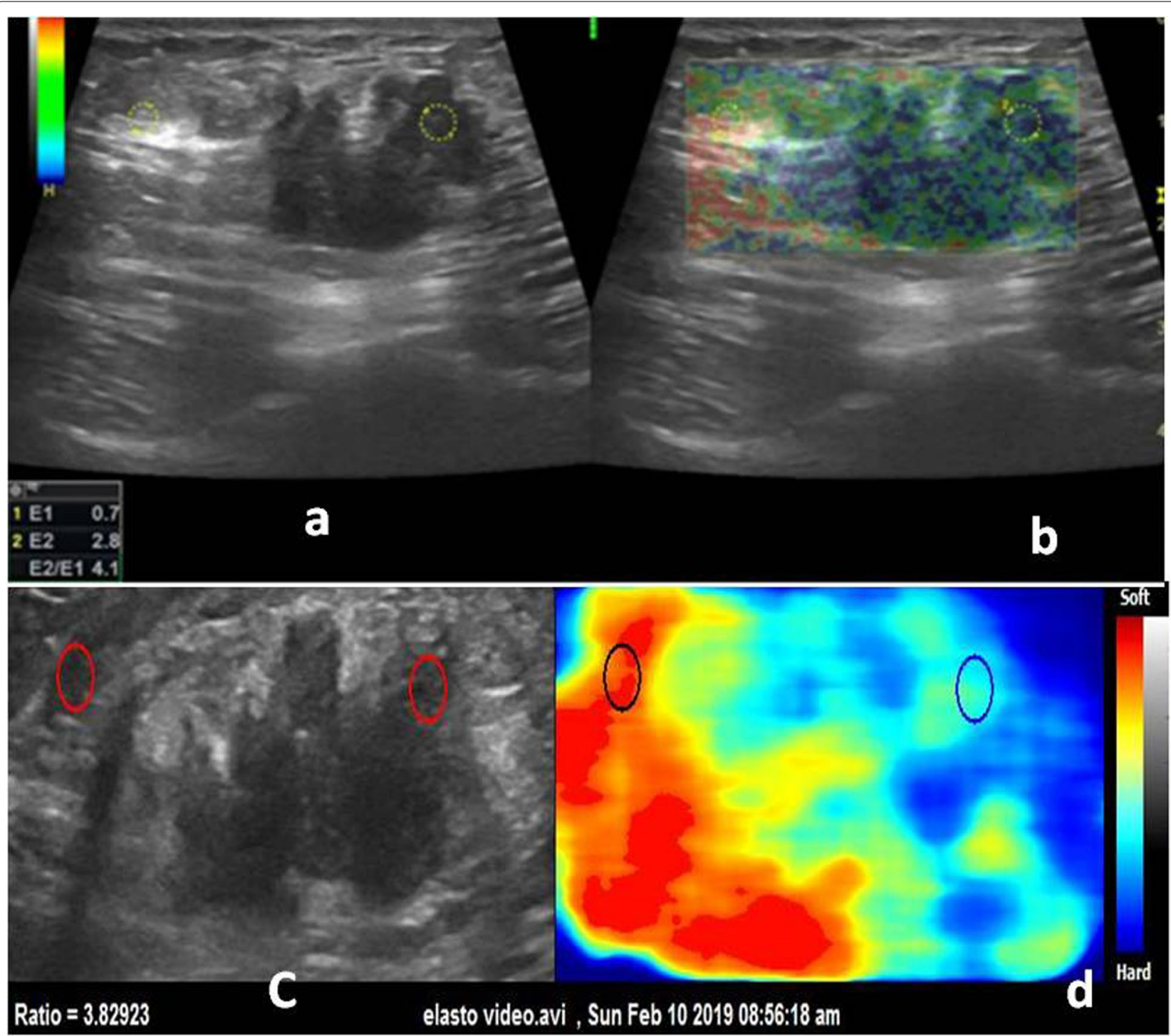

Fig. 250 year- old patient presented by a lump in the right breast, Ultrasound revealed a speculated mass lesion at right breast UOQ. The strain ratio calculated by conventional elastography was $4.1(\mathbf{a}, \mathbf{b})$. The strain ratio calculated by an Al-enabled elastography software was $3.8(\mathbf{c}, \mathbf{d})$. The final diagnosis was Invasive duct carcinoma grade II

or close follow-up,13 lesions were true positives, 12 were false positives, 15 lesions were false negatives and 13 were true negatives. conventional SR had a sensitivity of $46.43 \%$, a specificity of $52 \%$, a positive predictive value of $52 \%$, a negative predictive value of $46.43 \%$.

There are several factors which may affect the sensitivity and the specificity for example; the pathological distribution as well as the size and the depth of the lesions.

All the false-negative lesions (15 lesions) had SR below our cut-off point (2.4). They included one invasive tubular carcinoma grade I, one malignant papillary lesion, two mucinous carcinomas (Fig. 4), eleven IDC grade III.
As all the sono-elastograhic findings were correlated with the pathological results; this explained the number of false positive and false negative lesions, where we found that invasive duct carcinomas of low and intermediate grades are usually hard owing to the associated desmoplastic reaction, while the high grade carcinomas are usually less stiff as they may undergo necrosis with fat growth along with less desmoplastic reaction. Also we observed that papillary and mucinous carcinomas are usually soft lesions on elastography as they show cystic components or mucin on histology. And so this explained the number of false negative lesions we had in our study. 


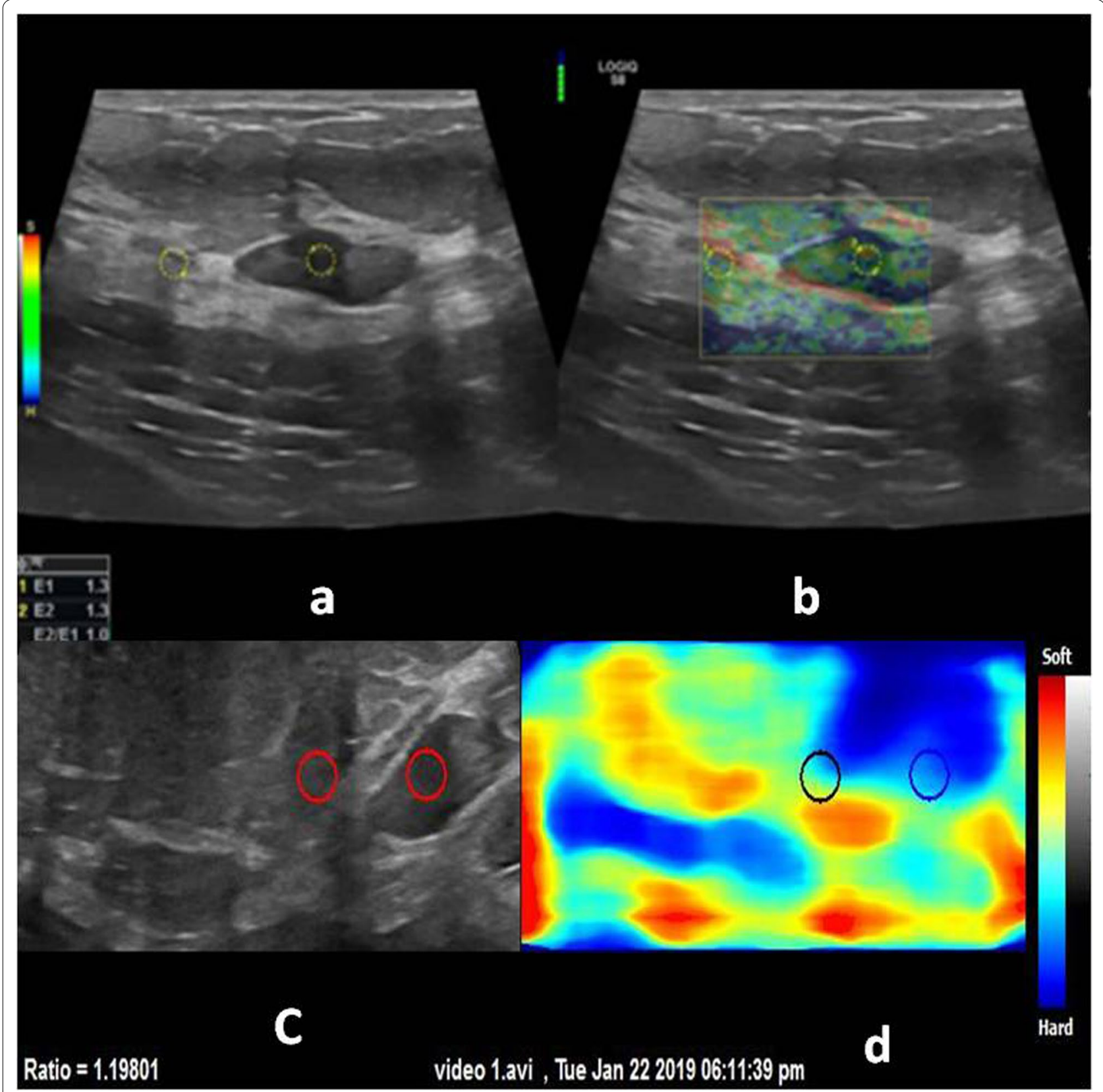

Fig. 345 year- old patient presented by a lump in the right breast, Ultrasound revealed a benign-looking mass lesion in the right breast. The strain ratio calculated by conventional elastography was $1(\mathbf{a}, \mathbf{b})$. The strain ratio calculated by an Al-enabled elastography software was $1.19(\mathbf{c}, \mathbf{d})$. The final diagnosis was a fibroadenoma

Meanwhile; all the false-positive lesions (12 lesions) had SR higher than our cut-off point. They included (3) fat necrosis lesions as confirmed by the result of core biopsy, (1) chronic abscess as confirmed by the result of core biopsy, (1) lesion was diagnosed as lipoma by the ultrasound features and on follow up, (7) lesions were fibroadenoma.
Similarly on correlation of the sonoelastographic findings with the pathological diagnoses, we found that although most fibroadenomata shows soft character, some of them are stiff due to hyaline degeneration or calcification or being large in size and this applies on the (7) lesions of fibroadenomata postulated in our study. 


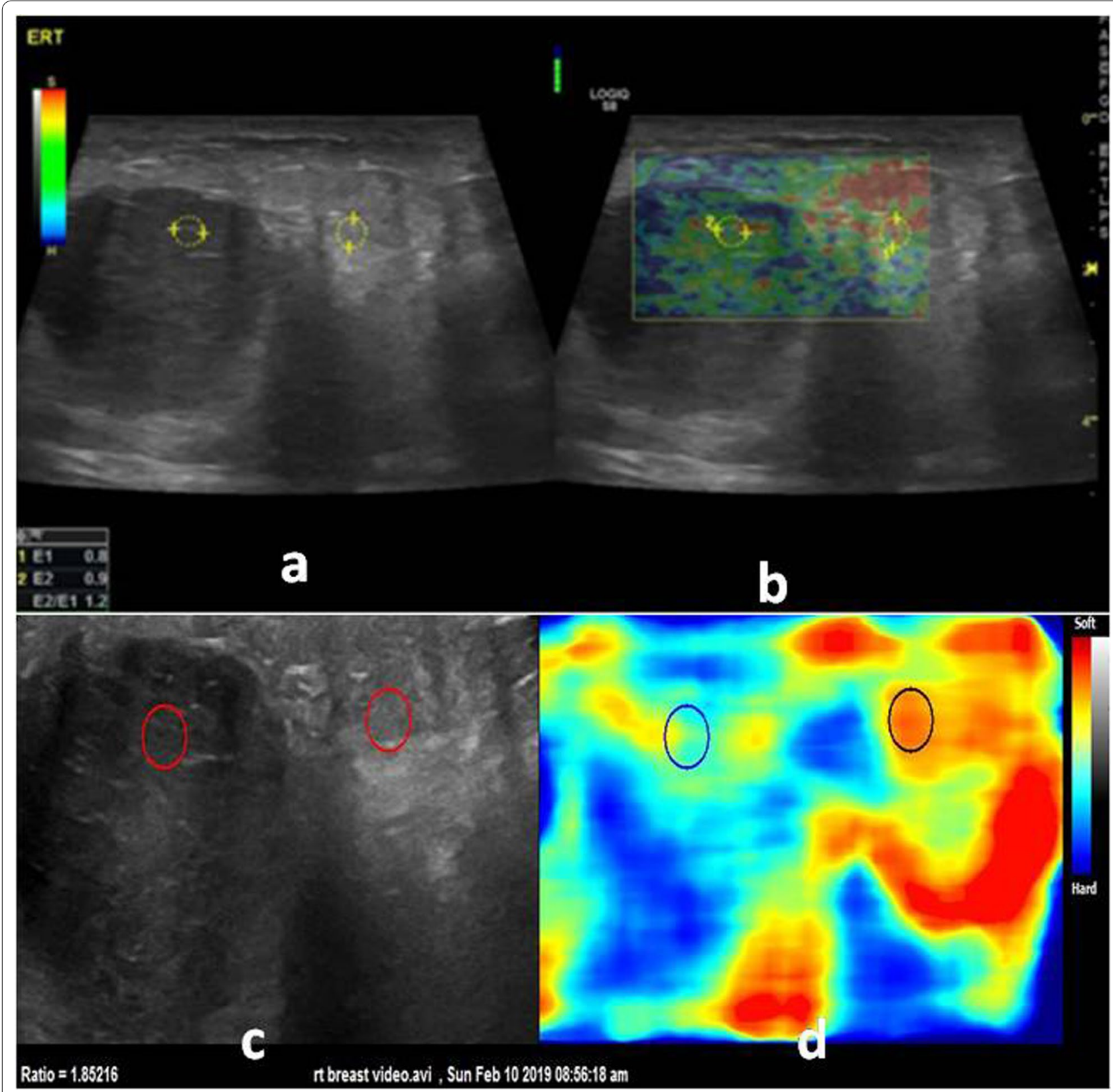

Fig. 435 year- old patient presented by a lump in the right breast, Ultrasound revealed a suspicious mass lesion at right breast LIQQ.The strain ratio calculated by conventional elastography was $1.2(\mathbf{a}, \mathbf{b})$. The strain ratio calculated by an Al-enabled elastography software was $1.8(\mathbf{c}, \mathbf{d})$. The final diagnosis was mucinous carcinoma

We found that large size of lesions may lead to falsepositive or false-negative results. Also deeply seated soft lesions may give false high results as they are compressed against the chest wall/ribs.

We assessed the advanced intelligent elastography software SR for each lesion (Figs. 2, 3). According to the histopathology results or close follow-up;17 lesions were true positives, 9 were false positives, 11 lesions were false negatives and 16 were true negatives. The calculated Advanced intelligent elastography software strain ratio sensitivity, specificity, positive likelihood, negative likelihood, positive predictive value, and negative predictive value were $60.71 \%, 64 \%, 1.6,0.6,65.4 \%$, $59.3 \%$ respectively.

All the false-negative lesions (11 lesions) had SR below our cut-off point (2.28). They included one invasive tubular carcinoma grade I, one malignant papillary, one lesion mucinous carcinoma, eight IDC grade III. 
All the false-positive lesions (9 lesions) had SR higher than our cut-off point. They included two fat necrosis lesions as confirmed by the result of core biopsy, one chronic abscess as confirmed by the result of core biopsy, six lesions were fibroadenoma.

In general, the histopathology/elastography results discordance owed to the same previously mentioned reasons in addition to that this novel software which needs special training.

In the current study, the strain ratio of malignant lesions was higher than that of benign ones, but the overlap is still existing so false-negative and false-positive results were unavoidable.

One of the important findings was that there was a moderate correlation between the SR calculated by the conventional elastography software (GE) and AI-elastography software. In addition, AI-Elasto SR showed better results than conventional SR. The specificity and positive predictive values are better which means it could exclude negative cases better. The sensitivity and negative predictive value are better which means it could confirm positive cases better. However, the test alone could not replace the pathology.

\section{Limitation of the study}

The major shortcoming of conventional elastography is that the extent of tissue compression influences the elasticity image and SR.

Inter and intraobserver variability may affect the final diagnosis. Intraobserver variability may be decreased by training, while interobserver variability needs further studies to be performed to get more standardized results and techniques.

Regarding limitations to the AI-elastography software, it needs special training. Also, no real-time results are available thus it needs post-processing which may be time-consuming. In addition, there is no compression indicator so inadequate compression may be a challenging problem.

\section{Conclusions}

The AI-enabled elastography software shows promising results compared to the conventional US elastography, yet further improvement and updates are needed. Elastography does not have the potential to replace conventional B-mode US for the detection of breast cancer but may complement the conventional US to improve diagnostic performance.

\footnotetext{
Abbreviations

Al: Advanced intelligency; BI-RADS: Breast Imaging -Reporting and Data System; MHz: Mega Hertz; ROI: Region of interest; ROC: Receiver-operating characteristic; SR: Strain ratio; US: Ultrasound.
}

\section{Acknowledgements}

The Al-enabled elastography software (US-Elasto) was supported by the Egyptian Information Technology Industry Development Agency (ITIDA) under ITAC Program CFP \#131 and DilenyTechnologies and Biomedical Engineering (DilenyTech) LLC.

\section{Authors' contributions}

RK, EK and MH wrote the manuscript. MM collected patient data and performed the statistical analysis. AM conceived of the study and blindly processed the Cine-loops of breast ultrasound using the Al-enabled elastography software. EF and $\mathrm{MH}$ participated in the design of the study. $\mathrm{MH}$ is responsible for correspondence to journal. All authors read and approved the final manuscript.

\section{Funding}

No funding sources.

\section{Availability of data and materials}

The datasets used and analysed during the current study are available from the corresponding author on reasonable request.

\section{Declarations}

\section{Ethics approval and consent to participate}

The study was approved by the ethical committee of faculty of medicine, Cairo University, the ethical approval number is not available.An informed written consent was taken from all subjects.

\section{Consent for publication}

All patients included in this research gave written consent to publish the data contained within this study.

\section{Competing interests}

Ahmed M. Mahmoud is innovation director at Dileny Technologies and Biomedical Eng LLC. No financial or non-financial competing interests for other authors.

\section{Author details}

${ }^{1}$ Department of Diagnostic and Interventional Radiology, Faculty of Medicine, Cairo University, 1 kasr Elainy street Fom El kalig, 52 Tayaran Street, Nasr City Cairo, Nasr City 00202, Egypt. ${ }^{2}$ Department of Biomedical Engineering, Cairo University, Giza, Egypt. ${ }^{3}$ Dileny Technologies and Biomedical Eng. LLC, Giza, Egypt.

Received: 13 August 2021 Accepted: 14 January 2022

Published online: 02 February 2022

\section{References}

1. Ghoncheh M, Pournamdar Z, Salehiniya H (2016) Incidence and mortality and epidemiology of breast cancer in the world. Asian Pac J Cancer Prev 17(S3):43-46. https://doi.org/10.7314/apjcp.2016.17.s3.43

2. Ricci P, Maggini E, Mancuso E, Maldur V et al (2017) Elastography in the diagnosis of breast lesions: comparison of different elastographic features. Acta Radiol 58(10):1189-1197. https://doi.org/10.1177/0284185116 687169

3. Seo M, Ahn HS, Park SH et al (2018) Comparison and combination of strain and shear wave elastography of breast masses for differentiation of benign and malignant lesions by quantitative assessment: preliminary study. J Ultrasound Med 37(1):99-109. https://doi.org/10.1002/jum.14309

4. Spak DA, Plaxco JS, Santiago L, Dryden MJ, Dogan BE (2017) BI-RADS ${ }^{\circledR}$ fifth edition: a summary of changes. Diagn Interv Imaging 98(3):179-190. https://doi.org/10.1016/j.diii.2017.01.001

5. Huang L, Ma M, Du Z et al (2019) Quantitative evaluation of tissue stiffness around lesion by sound touch elastography in the diagnosis of benign and malignant breast lesions. PLoS ONE 14(7):e0219943. https:// doi.org/10.1371/journal.pone.0219943 
6. Dietrich CF, Barr RG, Farrokh A et al (2017) Strain elastography —how to do it. Ultrasound Int Open 3(4):E137-E149. https://doi.org/10. 1055/s-0043-119412

7. Hiina T, Nightingale KR, Palmeri ML et al (2015) WFUMB guidelines and recommendations for clinical use of ultrasound elastography: part 1: basic principles and terminology. Ultrasound Med Biol 41(5):1126-1147. https://doi.org/10.1016/j.ultrasmedbio.2015.03.009

8. Ali MT, Mahmoud AM (2015) Elastography reconstruction from ultrasound brightness mode imaging using hierarchy recursive tracking. Annual Meeting Biomedical Engineering Society, Tampa, FL, USA

9. Salah MM, Sayed TA, Mahmoud AM (2015) A manufacturer independent ultrasound elastography add-on module. International Tissue Elasticity Conference, Bardolino, Italy

10. Mahmoud AME, Ali MTM (2020) Method and apparatus to measure tissue displacement and strain. (U.S. patent No. US10675002B2). U.S. Patent and Trademark Office

11. Akram M, lqbal M, Daniyal M et al (2017) Awareness and current knowledge of breast cancer. Biol Res 50(1):33. https://doi.org/10.1186/ s40659-017-0140-9

12. Azzam H, Kamal RM, Hanafy MM et al (2020) Comparative study between contrast-enhanced mammography, tomosynthesis, and breast ultrasound as complementary techniques to mammography in dense breast parenchyma. Egypt J Radiol Nucl Med 51:148. https://doi.org/10.1186/ s43055-020-00268-1

13. Gupta K, Sandhu P, Arora S et al (2018) Role of high resolution ultrasound complementary to digital mammography. Ann Afr Med 17(3):117-124. https://doi.org/10.4103/aam.aam_36_17

\section{Publisher's Note}

Springer Nature remains neutral with regard to jurisdictional claims in published maps and institutional affiliations.

\section{Submit your manuscript to a SpringerOpen ${ }^{\circ}$ journal and benefit from:}

- Convenient online submission

- Rigorous peer review

- Open access: articles freely available online

- High visibility within the field

- Retaining the copyright to your article 